\title{
I MONACI DI FRONTE ALLA MALATTIA FISICA E SPIRITUALE
}

\section{ROBERTO GRECI}

UDC: $27-788: 616-083 " 653 "$
27-42"653"

Original scientific paper

Manuscript received: 10. 12. 2016.

Revised manuscript accepted: 16. 01. 2017.

DOI: 10.1484/J.HAM.5.113737
R. Greci

Università degli Studi di Parma

Parma, Italia

Roberto.greci@unipr.it

The speech focuses on how disease and care are perceived and lived inside the monastic tradition. The sources, which have been used (hagiographies and monastic rules), show how in eastern monasticism, hermits and anchorites accept physical illness as a tool to reach the individual ascetic perfection, while the complex organisation of the coenobitic life introduces "diagnosis" as a new practice in order to distinguish the physical disease from the spiritual illness, and to uncover simulations. As a matter of fact, some monks pretended to be sick in order to avoid work and prayer spreading envy and disappointment inside the community. Therefore, it was necessary to create a designated room, the infirmary, not only in order to treat sick monks, but also to separate them from the others. Starting with S. Benedict, western monasticism, influenced by the Basilian tradition, accepted this kind of organisation and accorded a greater value to the care of the sick brothers, recognizing in this loving attention the spiritual enrichment of the monks (both the sick and the healthy ones) and of the whole of the monastic community. The custom of Cluny, moving from this theological and humanistic conception of care, includes disease and its spaces within the complex rites, whose forms appear as a severe anticipation of death.

Keywords: Middle Ages, monasticism, disease, hagiographies, monastic rules

Nella cultura monastica medievale, i termini malattia fisica e malattia spirituale, sono termini che ovviamente non rimandano a concetti condizionati dal sapere scientifico moderno. Nella prima categoria (malattia fisica) è possibile operare una distinzione tra aegritudo (l'infermità che costringe a letto) e debilitas o imbecillitas (concetti che i testi estendono ancheall'infantia ealla senectus), mentre più problematica è la definizione della seconda categoria (malattia spirituale o dell'anima) che si affaccia frequentemente già nei primi testi monastici indicando forme di disordine o di turbamento che danno luogo a varie tipologie di sofferenze. Se in quest'ultimo caso le manifestazioni più gravi (che le fonti monastiche attribuiscono per lo più ai non-monaci) richiedono un intervento miracolistico o un esorcismo (è il caso della possessione demoniaca), il semplice disagio interiore è in sé bivalente: può determinare infatti - positivamente - una spinta alla vita ascetica, ma può indurre, di contro, anche al peccato, compromettere cioè la salute dell'anima del monaco, mettere in crisi la sua scelta di vita e diventare anche una vera e propria malattia: per questo disturbo sono dunque necessari rimedi, ma si tratta di rimedi più sottili di quelli forniti dalla scienza mondana, con la quale peraltro l'ambiente monastico intrattiene variabili rapporti, spesso impotente: essi competono più al monaco esperto, al padre spirituale, all'abate, che non al medico. A volte queste malattie escono dalla dimensione spirituale determinando ingannevoli sofferenze fisiche (febbri, debolezza, freddo, mal di testa) ma più spesso determinano disturbi che potremmo definire piscologici: noia, svogliatezza, sfinimento, tristezza e infine accidia, la quale induce progressivamente il monaco a rifuggire dalla preghiera e dagli altri obblighi della vita monastica'. Una "malattia" strana, quest'ultima, radicata nelle stesse condizioni della vita monastica, sia nella sua dimensione ascetica sia nella sua dimensione comunitaria, nella quale si ravvisa tuttavia una buona dose di responsabilità del soggetto, soprattutto quando essa sconfina - e la cosa pare essere frequente - nella simulazione. Rispetto a questi disturbi, il confine tra l'intervento curativo della guida spirituale e quello del medico diventa labile e produce sovrapposizioni. D’altronde queste interferenze dipendono anche dal fatto che nella concezione cristiana l'uomo è percepito nella sua duplice, ma fortemente organica dimensione, fisica e spirituale insieme.

In questa sede si vuole discutere il peso riservato alla malattia e alla cura nelle fonti monastiche, non trascurando $i$ luoghi ad esse riservati; ovviamente non mi addentrerò nella ricostruzione dei luoghi e della materialità delle cure, prima di tutto perché si tratta di questioni che ad altri competono e in secondo luogo perché le fonti di cui mi servirò, cioè testi agiografici e regole, e i tempi cui esse si riferiscono (non mi spingerò oltre l'XI secolo) restituiscono scarse informazioni in proposito. L'intenzione è quella di affrontare il tema sia nella fase fondativa del monachesimo orientale (ascetico e cenobitico) sia nel processo di radicamento, di sviluppo, di uniformazione della esperienza cenobitica in Occidente. Mi pare infatti che norme sulla malattia e racconto della malattia possano contribuire a sottolineare ancora una volta la portata della rottura e dell'innovazione della cultura monastica cristiana rispetto alla tradizione culturale antica, e a scongiurare la tendenza a considerare il modello monastico come eccessivamente uniforme.

\footnotetext{
${ }^{1}$ Un'analisi della tristitia, una passio perniciosissima che produce instabilità della salute è in Cassiano, De coenobiorum institutis libri duodecim, in Patrologia latina $[=$ PL], 49, coll. 53-476, libro IX, col. 352 sgg. Ad essa affine è l'accidia, di cui Cassiano parla nel libro X che produce malattia dell'anima (ma anche disturbi fisici) e che assale soprattutto gli eremiti solitari (ibidem, col. 359 sgg.). Il rischio dell'accidia sarà presente nella Regola di Benedetto e resterà tema ricorrente nella letteratura ascetica che continuerà a collegarlo all'otiositas; cfr. J. LECLERCQ, Otia monastica. Études sur le vocabulaire de la contemplation au Moyen Âge, Roma 1963 (Studia anselmiana, 51), p. 41, 70 sgg. A tal proposito è eloquente la percezione del rischio dell'accidia in Pier Damiani che ad essa oppone, come rimedio, il recupero dell'otium letterario: vd. G. TABACCO, Pier Damiani fra edonismo letterario e violenza ascetica, in ID., Spiritualità e cultura nel Medioevo. Dodici percorsi nei territori del potere e della fede, Napoli 1993, p. 249-266.
} 
Le prime testimonianze di ambito orientale restituiscono una diffusa consuetudine di rapporti tra il monaco e la malattia sia nel contesto eremitico sia nel contesto cenobitico e una preoccupazione costante di collegare la crisi che l'infermità genera alla tensione verso la perfezione dell'ideale religioso. Ne è prova immediata il costante e ripetuto riferimento al precetto evangelico (Matteo, XXV, 36: infirmus, et visitastis me), che fa sì che la malattia venga percepita nei suoi significati morali, con una attenzione prevalentemente rivolta alle persone, più che alla malattia in sé. Nelle fonti agiografiche, nelle vite dei monaci, la malattia è una esperienza importante sia per il monaco che la subisce sia per il monaco che cura, entrambi impegnati nella lotta ascetica. Manca, quindi, lo sforzo di descrizione e di classificazione delle patologie e della loro eziologia, nonché il riferimento a forme specifiche di cura che esulino da questo obiettivo. Altra impressione che si ricava dalla lettura di queste fonti è quella di una scarsa importanza attribuita al corpo, cui (soprattutto da parte di chi abbraccia il monachesimo eremitico) sono riservate aspre privazioni e mortificazioni. In realtà è più corretto dire che l'importanza del corpo risiede nel suo essere mezzo e non fine e che la malattia fisica è, per chi la subisce, una delle tante mortificazioni utili all'ascesi, mentre per la persona sana vicina al malato (discepolo o confratello) essa diventa un'occasione di perfezionamento spirituale. Entro questo orizzonte di pensiero la malattia è vista come strumento provvidenziale riservato ai monaci (tanto malati quanto sani) per raggiungere (pur da due forme diverse di esperienza) il fine ultimo, che consiste appunto nella salute dell'anima e nella salvezza.

Proprio per questo già ad Antonio, o meglio agli scritti a lui attribuiti, risalgono poche semplici norme di assistenza e di comportamento indicate come prioritarie nell'organizzazione della giornata del monaco, nel rispetto dello status spirituale del malato a cui non va rinfacciata la sua infermità ${ }^{2}$. Se poi passiamo al testo agiografico (e il riferimento è alla Vita di Antonio di Atanasio, destinata a diventare un modello indiscusso e perennemente valido di vita monastica) la malattia diventa banco di prova e testimonianza palese del livello di perfezione ascetica raggiunto dal monaco, sia di quello che subisce l'infermità, sia di colui che guarisce il malato. Quest'ultimo infatti, guarendo, mostra di potersi porre come mediatore della volontà del Salvatore ${ }^{3}$, soprat- tutto nei confronti di chi ha più bisogno e cioè di chi non è monaco e che, in quanto tale, è più esposto alla malattia sia fisica che spirituale, spesso causata da una vita non ordinata ${ }^{4}$. Possiamo dunque dire che regola e agiografia sono i mezzi, diversi per destinatari ma equivalenti, per suggerire come affrontare la malattia e dominarla; gli strumenti proposti sono da un lato la serena e coraggiosa sopportazione (da parte del malato) e dall'altro una pluralità di interventi (del guaritore) che vanno dal miracolo e dall'esorcismo, alla indicazione di norme di vita salutari o semplicemente al sostegno spirituale e assistenziale. Che la narrazione agiografica stessa si ponga in ogni caso, indipendentemente dall'esito dell'infermità, come vera e propria medicina (soprattutto dello spirito ma, nei termini di cui si è detto, anche del corpo), è testimonianza un passo del prologo delle Vite dei monaci siri di Teodoreto di Cirro, che dichiara questa equipollenza e che attribuisce al racconto agiografico un valore perfino preventivo che marcia di pari passo con una debole fiducia nell'arte del medico, la cui arte era, al tempo, pur ampiamente diffusa ${ }^{5}$. L'agiografo, infatti, ha il merito di tramandare memoria di uomini superiori ai medici; questi ultimi spesso falliscono, prescrivono una medicina per ogni singola malattia, mentre il monaco guaritore, la cui memoria è preservata dal racconto agiografico, dispensa, tramite la preghiera, un rimedio per tutte le malattie, sia del corpo sia dell'anima. Rispetto al malato, il narratore ha il merito di custodire il ricordo vivo di uomini che, con la forza degli atleti, hanno esemplarmente mostrato impassibilità e hanno emulato la natura incorporea superando ogni limite materiale ${ }^{6}$.

Soprattutto Atanasio, ancora una volta nella Vita di Antonio, punta a costruire un modello di rapporto tra monaco e malattia radicalmente fondato sulla fede, che sancisce, anche sotto questo aspetto, la superiorità della vita monastica di per sé foriera di salute: l'astensione dai cibi, dai cattivi pensieri e dai piaceri della carne, scelte radicalmente alternative rispetto agli stili di vita correnti, stanno alla base della perfetta salute di Antonio e come tali sono più o meno esplicitamente raccomandati, come una medicina preventiva, ai monaci ${ }^{7}$. La narrazione agiografica è la prova provata di tutto questo, poiché soddisfa il bisogno di certezze degli increduli nei confronti dei quali non si esita a ricorrere alla forza probatoria e autoritativa dello scritto ${ }^{8}$. Assistiamo in questo caso a un confronto serrato tra la cultura diffusa del

\footnotetext{
${ }^{2}$ S. Antonii abbatis Regulae ac praecepta ad filios suos monachos, PL, 103, coll. 423-428, cap. 5 (cum autem surrexeris mane singulis diebus, inquire de aegrotis qui apud te sunt) e 34 (ne obstringas aegrotum ad manducandum, nec retrahas ab illo cibum, ne conturbes animam illius afflictam). Per questo antico testo non attribuibile ad Antonio, vd. G. TURBESSI, Regole monastiche antiche, Roma 1978, p. $17 \mathrm{sgg}$.

${ }^{3}$ Vita di Antonio, a cura di G.J.M. Bartelink, in Vite dei santi, I, a cura di Ch. Mohrmann, Milano 1987, p. 115: Antonio esortava i malati a sopportare pazientemente e a comprendere che la guarigione miracolosa non era opera sua né di nessun altro uomo, ma solo del Signore che fa quando vuole e a chi vuole. I malati accoglievano i discorsi del vecchio come una medicina e imparavano a sopportare con pazienza e a non disperarsi.

${ }^{4}$ Alludiamo alla guarigione di una vergine di Busiride di Tripoli che aveva malattie pessimas et valde miserabiles: le lacrime e il muco e gli umori che uscivano dalle orecchie della ragazza cadevano a terra e immediatamente diventavano vermi; inoltre corpus ipsius passum erat paralysin et oculi non erant in proprietatem (ibidem, cap. 58, p. 117). Di malattia mentale è afflitto invece un illustre giovane uomo posseduto da un daemon pessimus che lo portava a mangiarsi le dita; la guarigione fu possibile poiché Antonio, mosso a pietà, pro iuvene orabat et nocte vigliabat cum ipso (ibidem, p. 124).

5 «Come, infatti, coloro che si crede curino il corpo preparano medicine per combattere le malattie e soccorrere i malati, così la fatica di narrare queste storie diviene, per così dire, una medicina che allontana le malattie»; TEODORETO DI CIRRO, Storie di monaci siri, Roma 1995, p. 66 (Prologo).

${ }^{6}$ Ibidem, p. 200 (Marone).

7 Vita di Antonio, op. cit. (n. 3), p. 175 sgg., Antonio ebbe un'ottima salute: anche da vecchio aveva occhi sani e perfetti, tutti i denti, sano di piedi e di mani, raggiante e più robusto di coloro che mangiano cibi vari, fanno bagni, vestono abiti diversi. In questo senso possiamo leggere i consigli forniti dalla beata Melania al diacono Evagrio del Ponto, colpito da febbri che i medici non riescono a guarire. Melania dice che la malattia, conseguente ad una vita sregolata, non è estranea a Dio (athées) e indica al malato la scelta della vita eremitica; cfr. PALLADIO, La storia lausiaca, a c. di J.M. Bartelink, in Vite dei Santi, a c. di C. Mohrmann, II, Milano 1974, p. 199 (Evagrio).

${ }^{8}$ Vita di Antonio, op. cit. (n. 3), p. 123. La coincidenza (intesa come rapporto di causa ed effetto) tra preghiera e guarigione ad opera di Antonio è attestata da una nota scritta da Archelao, un funzionario che aveva chiesto al santo di guarire una ragazza estenuata dall'eccesso di astinenza che le provocava dolori
} 
tempo, che per quanto permeata di contenuti sacrali aveva la possibilità di ricorrere alla medicina "scientifica" e ai suoi rimedi - di fatto inefficaci soprattutto nel caso di infermità che oggi definiremmo psichiche - e la cultura del monaco che invece mostra, nei confronti di chi è affetto da tali disturbi, una comprensione e una pietà sconosciute perfino a coloro che erano affettivamente vicini agli ammalati9.

In realtà questi primi testi, permeati da una visione religiosamente radicale della malattia e della guarigione, non mancano di registrare anche situazioni diverse da quelle più frequentemente narrate e talora perfino contrastanti con esse. Emerge infatti, talora, un interesse non occasionale del monaco per una platea di ammalati ben più vasta di quella, pure non disprezzabile, costituita da coloro che volontariamente e fiduciosamente si recano alla sua cella imponendogli visite non ricercate, cui l'asceta fa fronte solo oppure con l'aiuto dei suoi più stretti confratelli e discepoli ${ }^{10}$. Ciò può avvenire quando il monaco, privilegiando una vita attiva, abbandona la solitudine ed entra in contatto con le istituzioni ecclesiastiche preposteall'assistenza (un'assistenza ovviamente assai meno personalizzata) nell'indistinto ambiente urbano" ${ }^{\text {. }}$ Tale contatto rischia di determinare oscillazioni nella valutazione sia della malattia sia della cultura e dei rimedi offerti dalla medicina. Si può registrare infatti l'eco di un vero e proprio scontro tra l'ideale monastico più severo e le consuetudini del tempo. Questa contrapposizione è evidente nella Vita di Macrina scritta da suo fratello, il monaco Gregorio di Nissa (335-395) ${ }^{12}$, ovvero nel contrasto tra la giovane, che pur essendo gravemente malata ritiene la malattia una prova mandata dall'alto, meritata e quindi sopportata con pazienza ${ }^{13}$, e la madre preoccupata per la sua salute. Macrina punta a forme di ascesi estrema, rifiutando le cure mediche e privilegiando la fiducia assoluta in Dio, mentre la madre, invece, prospetta una possibilità di accordo tra cultura medica e cristianesimo, ritenendo la medicina un'arte insegnata da Dio per la salute degli uo- mini, quindi non incompatibile con la professione di fede. La posizione della madre di Macrina era peraltro condivisa da figure esemplari di monaci. Il disprezzo per il corpo è decisamente contrastato, ad esempio, dallo stesso Gregorio di Nissa, che, non a caso vescovo e monaco nel contempo, condivide, contro le idee della sorella di cui narra la vita, il parere della madre Emmelia, ma anche da Basilio che non esita a proporre più moderate concezioni nella sua Regola ${ }^{14}$. D’altronde, per rimanere sempre entro i testi agiografici, sappiamo che lo stesso Palladio, dimorando nel deserto e ammalatosi di idropisia, non si rifiuterà, seguendo i consigli dei confratelli, di recarsi ad Alessandria per curarsi. Qui i medici lo inviteranno a trasferirsi in Palestina per godere del clima migliore di quella regione che Palladio, nel rispetto del parere dei medici, raggiungerà immediatamente ${ }^{15}$. Anche Macedonio, che vive sui monti in solitudine, esorta con forza la madre di Teodoreto di Cirro, indebolitasi fino allo stremo per la severa vita ascetica, per i digiuni e per il rifiuto di ogni cura medica, a nutrirsi di cibi idonei per riprendersi. L'argomentazione di Teodoreto, anch'egli vescovo e monaco, è che la prostrazione fisica conseguente al digiuno, spia di un desiderio di abbreviare la vita con le sue fatiche, deve essere valutata addirittura come una dimostrazione di poco coraggio, inammissibile per l'asceta/atleta, un vero e proprio peccato $^{16}$.

Teodoreto non manca di mettere in pratica queste idee. Di ciò ci informa narrando della vita esemplare di Giacomo, un suo contemporaneo allievo di Marone, che vive in una montagna vicino a Cirro privo di ogni riparo e che, afflitto da una malattia intestinale (flusso di bile), non solo rifiuta le cure, ma non cessa di sottoporsi a pesanti autopunizioni, tormentando il proprio corpo con ferri e catene e esponendosi al sole impietoso: pratiche che, dopo un temporaneo miglioramento, lo condurranno alla morte. Teodoreto corre da lui per assisterlo e per moderare il suo ostinato atteggiamento. Il suo tentativo, tuttavia, è destinato al fallimento, poiché

\footnotetext{
allo stomaco e a un fianco: «Archelao prese nota del giorno in cui aveva pregato e giunto a Laodicea trovò la vergine guarita. Chiese l'ora e il giorno e venne a sapere che era il giorno in cui Antonio aveva pregato. Tirò fuori la carta e la mostrò a tutti e tutti ascoltando il fatto furono presi da meraviglia poiché il Signore l'aveva guarita quando Antonio aveva invocato la sua bontà».

9 Ibidem, cap. 64, p. 125. Un uomo illustre, tormentato dal demonio dal quale era spinto perfino a mangiare i propri escrementi, viene condotto, fuori di sé, al cospetto del santo. I familiari chiedono ad Antonio di pregare per lui. Antonio ha pietà del giovane e passa la notte in veglia per lui. All'alba il giovane si scaglia contro Antonio e lo percuote suscitando l'ira dei suoi accompagnatori. Antonio redarguisce questi ultimi perché comprende come il malato non abbia responsabilità del proprio comportamento («non adiratevi con lui; è il demonio che è in lui») e caccia il demonio che lo tormenta. Analogo l'episodio narrato nella vita di Macario l'Egiziano cui una madre porta al santo il figlio malato, ovvero posseduto dal demonio igneo; la bulimia di cui era affetto (così oggi definiremmo il disturbo) lo costringeva a mangiare tutto ciò che la madre gli preparava e in più i propri escrementi e a bere la propria orina. Macario lo accoglie e prega per lui. Dopo uno o due giorni il male si attenua; PALLADIO, op. cit. (n. 7), p. 77 (Macario l’Egiziano).

${ }^{10}$ Macario l'Egiziano vive con due discepoli, uno di questi conduce vita solitaria in una piccola cella vicina, mentre l'altro è sempre al suo fianco nell'interesse di quelli che vengono a farsi curare; ibidem, p. 71.

"Il monaco caritatevole che viene dalla vita militare, fattosi asceta per vent'anni, risiede presso il vescovo della città passa la vita aiutando gli altri, poveri e ricchi, in prigioni e ospedali. Come ovunque avviene, nel portico della chiesa cittadina soggiornano molti malati che chiedono elemosina, soli o sposati. Una notte la moglie di uno di questi partorisce e il monaco l'aiuta prendendo il posto della levatrice, mostrandosi insensibile al disgusto del parto. L'amore per gli uomini gli fa preferire il desiderio di assistere i bisognosi ai libri e alla lettura: ibidem, p. 281.

${ }^{12}$ GREGORIO DI NISSA, Vita di santa Macrina, a cura di E. Giannarelli, Milano 1997, p. 139 sgg.

${ }^{13}$ Macrina tutt'al più si cura con lacrime cadute per terra durante le sue orazioni e con esse si unge il seno afflitto da tumore o si fa segnare dalla madre Emmelia con una croce. Analogo atteggiamento ha l'asceta Beniamino, stimato per le guarigioni ottenute con l'imposizione delle mani, che però sopporta la propria idropisia con pazienza degna di Giobbe e conserva una gratitudine senza fine per la prova cui è sottoposto: cfr. PALLADIO, op. cit. (n. 7), p. 55 (Beniamino), in ottemperanza a quanto indicato da Paolo nella Lettera ai Corinzi, II, 12, 10 («mi compiaccio delle mie infermità ... quando sono debole allora sono più forte»).

${ }_{14}$ BASILIO, Regulae fisius tractatae, in Patrologia graeca [= PG], 31, p. 889-1052, a col. 1043-1044: Interrogatio LV (An medicinae usus pietatis instituto conveniat): alla domanda posta viene risposto l'arte medica, come l'agricoltura, l'arte tessile e quella delle costruzioni, «a totius nostrae vitae moderatore Deo concessa est».

${ }^{15}$ PALLADIO, op. cit. (n. 7), p. 175 (Giovanni di Licopoli),

${ }^{16}$ TEODORETO DI CIRRO, op.cit. (n. 5), p. 183-84 (Macedonio).
} 
Giacomo accetterà da lui solo una banale tisana raffreddata, bevuta fra l'altro con molta diffidenza, ad occhi chiusi, come si fa con le bevande amare e disgustose ${ }^{17}$. Diversamente da Giacomo, però, si comporta Stefano di Libia, asceta sulla palude della Mareotide, che non esita a sottoporsi perfino alle necessarie cure del chirurgo; va comunque detto che nello straziante rimedio Stefano più che i vantaggi della guarigione vede un'ulteriore occasione per dimostrare la propria impassibilità ascetica ${ }^{18}$. Pur nella varietà, le testimonianze restituiscono una prevalente omogeneità di atteggiamenti. Risulta comunque percepibile una diversità di posizioni tra chi, pur affiancando e alla fine scegliendo una vita monastica proviene da quella ecclesiastica e chi da subito ha compiuto una scelta esclusivamente e radicalmente ascetica.

Differenze sono inoltre percepibili tra il monaco anacoreta e il monaco inserito in una dimensione cenobitica. Severo con se stesso oltre ogni limite, Macario di Alessandria non esita neppure a rifiutarsi di accogliere e di guarire un presbitero fornicatore addetto ai servizi divini con la testa completamente corrosa dal cancro, perchél'asceta continua a ritenere che il male, nel caso specifico, sia una giusta punizione per una persona indegna di guarigion $\mathrm{e}^{19}$. Tale severità, che investiva anche le sue abitudini alimentari, non mancò di entrare in conflitto con la dimensione cenobitica della vita monastica dalla quale pure fu attratto. Quando infatti, ad un certo punto della sua vita, si recò in un monastero pacomiano della Tebaide mostrandosi pronto a lavorare come operaio con gli oltre mille uomini che in esso vivevano nel rispetto della regola, le sue consuetudini ascetiche e alimentari provocarono contestazioni nel monastero tanto che Pacomio, scoperta la sua identità, pur lodandolo, non esitò a pregarlo di ritornarsene alla sua vita solitaria ${ }^{20}$.

A conferma di ciò, possiamo ricordare Macario l'Egiziano, fondatore della comunità monastica di Scete e anche prete, dedito a forme di ascesi rivolte più alla contemplazione che alla macerazione del corpo e straordinariamente sensibile alle necessità dei malati che a lui si rivolgono; con grande umanità e pazienza li accompagna pazientemente alla guarigione. Proprio in relazione alle sofferenze di un giovane bulimico, non manca di suggerire alla madre dell'infermo un regime alimentare consono ai reali bisogni del figlio. E tali consigli, che contrastano abitudini diffuse (la madre tollerava che il figlio mangiasse 10 libbre di pane), sembrano ispirarsi alla moderazione e alla regolarità praticata nella comunità monastica, basata sul digiuno, sul lavoro, sul moderato soddisfacimento dei bisogni alimentari (3 libbre di pane anziché 10$)^{21}$.
Il discrimine tra atteggiamenti diversi sembra dipendere non solo dalla diversa sensibilità religiosa (e di conseguenza dal diverso modo di vivere la propria esperienza monastica) ma anche dalla formazione e dalla provenienza sociale del singolo. La vicenda di Eulogio è eloquente. Figura psicologicamente complessa di studioso umanista, ad un certo punto della sua giovane vita, annoiato di se stesso, sente l'impulso di isolarsi dal mondo. L'occasione per intraprendere il cammino ascetico viene proprio dall'incontro con la malattia o meglio con la menomazione. Incontrato in piazza un uomo privo di mani e piedi e quindi incapace di badare a se stesso, se lo porta in cella ripromettendosi di prendersene cura per tutta la vita. Dopo 15 anni di dedizione, sarà lo storpio che non tollererà più questa forzata convivenza e aggredirà Eulogio ribellandosi ad una intellettualistica strumentalizzazione della malattia prevalentemente funzionale all'interesse spirituale del benefattore. Solo il consiglio di Antonio, richiesto da Eulogio, ricomporrà il dissidio restituendo una dimensione paritaria al rapporto tra i due e sottolineando il fatto che la reciproca sopportazione avrebbe garantito ad entrambi la ricompensa divina ${ }^{22}$.

In buona sintesi possiamo dire che la maggior parte dei monaci asceti considerano il proprio corpo e la propria sofferenza fisica in modo diverso (più severo) dal corpo e dalla malattia del prossimo e in particolare di chi non è monaco, fornendo opportuni rimedi a chi soffre. Il rifiuto delle cure, quando non la ricerca volontaria della sofferenza, fa infatti parte della rinuncia del mondo e del superamento della corporeità, che non necessariamente costituiscono un obiettivo per chi vive nel mondo. Ma i rimedi forniti al prossimo non sono rimedi medici, specialmente se la malattia di cui si tratta è una malattia spirituale, una forma di disagio che solo secondariamente può trasformarsi in un disturbo fisico. Di fronte a questi tipi di malattia la religiosità e l'esperienza vissuta del monaco possono esibire tutta la loro efficacia. Nei confronti della malattia fisica, invece, il ricorso alla medicina è frequente e a volte accettato dallo stesso monaco. Questa maggiore disponibilità si affianca a forme di pratica ascetica più moderate e regolate, più consone alla vita cenobitica, una realtà affollata e variegata sotto ogni profilo, anche sociale e culturale, in cui diventa necessario smussare le difformità individuali e puntare ad una uniformità di comportamenti. In questo contesto può trovare dunque spazio anche la tradizione medica, laddove particolarmente sviluppata (ad esempio nei monasteri pacomiani dell'Egitto), e il sapere ippocratico-galenico (nei monasteri basiliani in Cappadocia). Proprio Basilio ad esempio, pur ritenendo che non

\footnotetext{
${ }_{17}$ Ibidem, p. 215, (Giacomo). Diverso Linneo che vive in un recinto dove Teodoreto lo va a trovare; afflitto da una dolorosa colica, si rotolava e contraeva le membra, ma non si giovò dell'arte medica né delle medicine, ma delle preghiere e del segno di croce; analogamente quando fu morso da vipera, ibidem, p. $235-236$ (Talassio o Linneo).

${ }^{18}$ PALLADIO, op. cit. (n. 7), p. 133 (Stefano di Libia): quando riceve la visita di alcuni discepoli di Ammonio e di Evagrio, Stefano, afflitto da ulcera cancrenosa nei testicoli e nel glande, è intento a sopportare stoicamente il dolore dell'amputazione senza smettere di lavorare e di parlare con gli ospiti ritenendo il male un castigo meritato, tollerato da Dio che opera solo per buoni fini. Dimostrando che la punizione in vita è migliore di quella eterna, edificò i suoi visitatori infondendo loro coraggio e fermezza.

${ }^{19}$ Ibidem, p. 95 (Macario d'Alessandria).

${ }^{20}$ Ibidem, p. 85. La incompatibilità tra ascetismo estremo e vita cenobitica è presente anche in Simeone, i cui digiuni durante una decennale esperienza comunitaria irritarono i compagni e i superiori che non esitavano a definire il suo zelo disordine; cfr. TEODORETO DI CIRRO, op. cit. (n. 5), p. 248 sgg. (Simeone)

${ }^{21}$ PALLADIO, op.cit. (n. 7), p. 77 (Macario l'Egiziano). In generale sul tema del pane nella dieta monastica, cfr. G. ARCHETTI, "Noli pane satiari". Il pane sulla mensa dei monaci, in La civiltà del pane. Storia, tecniche e simboli dal Mediterraneo all'Atlantico, Atti del Convegno internazionale di studio (Brescia, 1-6 dicembre 2014), a cura di G. Archetti, Spoleto-Milano 2015 (Centro studi longobardi. Ricerche, 1), pp. 1663-1704.

${ }^{22}$ PALLADIO, op.cit. (n. 7), p. 107 (Eulogio e lo storpio).
} 
tutte le infermità hanno cause naturali, ma sono piuttosto punizioni inflitte per il ravvedimento dei peccati, giudicava una ostinazione non ricorrere alla medicina in caso di bisogno. Non mancava in ogni caso di mettere in guardia da una fiducia eccessiva nei medici condannandone l'uso e l'abuso ${ }^{23}$. Con ciò la dimensione religiosa si ritagliava uno spazio di intervento non banalmente sovrapponibile a quello della scienza medica e una possibilità di controllo e di gestione di qualsiasi forma di malattia, che si esprime concretamente anche attraverso spazi appositamente riservati alla cura.

Certo vi sono differenze tra la soluzione praticata nella laura e quella praticata nel cenobio. Il più spinto individualismo del monaco che vive nella laura non lo distacca, in caso di malattia, dalla cella, in cui gli attori sono il monaco e i suoi pochi confratelli o discepoli che dispensano all'infermo cure semplici e palliative ${ }^{24}$. Solo in caso di malattia prolungata, si fa riferimento ad un ricovero nell'unico punto "centrale" del monastero: la chiesa. Invece, nel caso dell'organizzazione cenobitica, vediamo apparire uno spazio apposito, l'infermeria. Teniamo comunque presente che sebbene nei monasteri pacomiani vi fosse un'infermeria e sebbene vi fossero medici tra i monaci, le cure ivi dispensate erano estremamente semplici e gli infermieri erano pur sempre confratelli. Non siamo peraltro in grado di sapere se questo spazio fosse inizialmente una semplice cella adibita alla specifica funzione, oppure una realtà più complessa, simile ad esempio alle infermerie militari romane, che Pacomio doveva conoscere e che anche noi conosciamo (seppure in contesto non egiziano). Scarse sono le fonti archeologiche; certo è che nella Vita di Pacomio (324) sappiamo che questi aveva destinato una apposita casa per il conforto dei fratelli ammalati, governata da un oikiakos e da un deuteros, attestata peraltro anche nei monasteri di Shenoute e basiliani e comprensiva di una propria dispensa, cucina e refettorio ${ }^{25}$. I medici in essa operanti sono medici che hanno scelto di vivere dentro il monastero, come nel caso della laura di Nitria (500 monaci) dove un certo numero di dottori continua ad operare o come nel caso del cenobio di Shenoute, dove troviamo medici uomini e donne (queste ultime frequenti in Egitto) che risiedevano nel monastero tripartito articolato in due comunità maschili e in una femminile. Altrove, però, ritroviamo medici esterni che dalla città si recano nei monasteri, vicini o lontani, o nelle laure ${ }^{26}$. Nell'un caso e nell'altro dobbiamo desumere che le conoscenze mediche dell'epoca, diverse a seconda delle aree, penetravano nel monastero, anche se tali conoscenze, nel monastero, venivano di fatto private di autonomia.

Cercando di individuare modelli influenti su questa organizzazione monastica, non dobbiamo dimenticare il ruolo delle chiese deputate all'assistenza sulla base dei canoni del concilio di Nicea e quello dei ricoveri cittadini con funzioni plurime (xenodochi), ma anche con funzioni di veri e propri ospedali specializzati (nosokomeia), come quello per ciechi a Gerusalemme o quello che dispensava cure ostetriche ad Alessandria. L'esistenza di queste realtà fanno sì che nell'organizzazione dell'assistenza riservata ai confratelli entro il monastero non possiamo ravvisare qualcosa di totalmente inedito. In questo contesto orientale abbiamo perfino esempi di collaborazione tra monastero e xenodochi cittadini. È il caso della regola di Abramo di Kashkar, siro orientale, per un cenobio pacomiano organizzato come una lavra nella Persia di fine VI secolo (si tratta del monastero di Marde, sul monte Izla). In questo caso si prevedeva la possibilità per il malato di soggiornare in città, dietro permesso della comunità e limitatamente allo xenodochio, in quanto si voleva evitare che il monaco girovagasse o risiedesse in case private per non essere di peso a nessuno e per non dare scandalo ${ }^{27}$. La comparsa dell'infermeria monastica rappresenta insomma un riorientamento di esperienze conosciute, ispirato al bisogno di una autonomia indispensabile per raggiungere un'autosufficienza funzionale alla concezione del corpo e all'organizzazione interna di realtà numericamente cospicue.

Entro il monastero sono presenti figure che assomigliano a quelli che oggi potremmo definire infermieri. Se la loro esistenza indica il superamento della tradizionale dimensione familiare dell'assistenza, la loro funzione non presenta tuttavia caratteristiche "professionali". Nel sistema pacomiano essi sono inseriti in apposite unità e rispondono a compiti precisi, che restano tuttavia del tutto generici; chiamati a confortare i malati, a distribuire loro materassi, cuscini, abiti speciali e cibo, il loro incarico, assunto "a rotazione", si alterna ad altre funzioni utili alla vita della collettività. Il parere del medico, che interviene nei casi più gravi, è subordinato alle decisioni del preposto al quale il malato si rivolge per potere ricoverarsi in infermeria e che stabilisce terapia e dieta, nel rispetto della limitata credibilità - come si è detto - riservata alla medicina, ad esempio, nel pensiero basiliano $^{28}$. Data questa preminenza del ruolo del monastero nella gestione della malattia, sarebbe più opportuno parlare

\footnotetext{
${ }_{23}$ BASILIO, Regulae fisius tractatae (n. 14), Interrogatio LV, col. 1047: «Nam et in medicorum manibus sanitatis suae spem collocare, belluinum est». ${ }^{24}$ A.T. CRISLIP, From Monastery to Hospital. Christian Monasticism and the Transformation of Health Care in Late Antiquity, Ann Arbor 2005 , p. 12.

${ }_{25}$ Per le notizie che seguono, vd. ibidem, cap. I. Non abbiamo evidenze né per i monasteri dell'Asia minore, né per quelli dell'Africa settentrionale dove invece disponiamo delle fonti scritte relative ai monasteri di Basilio e di Agostino (IV secolo). Una infermeria monastica precoce (forse risalente a fine V) è stata scavata in Egitto (monastero di Apa Geremia a Saqqara), che in effetti si presenta come una delle più grandi strutture del cenobio, dal momento che occupava un terzo dello spazio riservato alla chiesa principale e che era di poco inferiore al refettorio. Per una esemplificazione di questo contesto egiziano, vd. anche P. GROSSMANN, D. BROOKS-HEDSTROM, M. ABDAL-RASSUL, E. BOLMAN, The Excavation in the Monastery of Apa Shenute (Dayr Anba Shinuda) at Suhag: With an Appendix on Documentary Photography at the Monasteries of Anba Shinuda and Anba Bishoi, Suhag, by Elisabeth S. Bolman, «Dumbarton Oaks Papers», 58 (2004), pp. 371-99.

${ }^{26}$ CRISLIP, op. cit. (n. 24), p. 14.

${ }^{27}$ P. DESEILLE, Il vangelo nel deserto. Un itinerario di spiritualità, Magnano (Bi) 2000, cap. 6.

${ }^{28} \mathrm{Vd}$. supra a nota 24. Sulla dieta monastica cfr. le esaurienti riflessioni di G. ARCHETTI, "Infundit vinum et oleum". Olio e vino nella tradizione monastica, in Olio e vino nell'alto medioevo, Spoleto, 20-26 aprile 2006, Spoleto 2007 (Settimane di studio della Fondazione Centro italiano di studi sull'alto medioevo, LIV), pp. 1099-1209; ID., "Vas optimo lacte plenum". Latte e formaggio nel mondo monastico, in La civiltà del latte. Fonti, simboli e prodotti dal Tardoantico al Novecento, Atti dell'incontro nazionale di studio (Brescia, 29-31 maggio 2008), a cura di G. Archetti, A. Baronio, Brescia 2011, pp. 249-278; ID., "Mensura victus constituere". Il cibo dei monaci tra Oriente ed Occidente, in L'alimentazione nell'alto medioevo: pratiche, simboli, ideologie, Sessantatreesima settimana di studio (Spoleto, 9-14 aprile 2015), Spoleto 2016, pp. 757-797; ID., I monaci a tavola: norme e consuetudini alimentari, in Gli spazi della vita comunitaria, Atti del Convegno internazionale (Roma-Subiaco, 8-10 giugno 2015), a cura di L. Pani Ermini, Spoleto 2016 (De re monastica, 5), pp. 305-327.
} 
non tanto di diagnosi ma piuttosto di diakrisis, l'operazione cioè di discernimento con cui vengono distinte le malattie vere da quelle false, le malattie del corpo (generate da cause naturali), da quelle dello spirito, più sfuggenti e talora confondibili con la malattia fisica, campo privilegiato del religioso esperto ${ }^{29}$. Le cause della grande attenzione riservata alla infermità dello spirito, che indirettamente comporta un controllo su tutte le altre forme di malattia, dipende dal fatto che essa è ritenuta riconducibile alla responsabilità individuale e pertanto, una volta riconosciuta come tale, diventa oggetto di azioni correttive e/o disciplinari tese soprattutto a non compromettere la scelta monastica e a riorientarla ${ }^{30}$.

La preoccupazione maggiore è quella di sventare le simulazioni, pericolose per la pacifica vita comunitaria, una preoccupazione destinata a diffondersi nelle regole monastiche, per approdare nella latina Regula Magistri ${ }^{3}$. Questa necessità primaria, che porta come si è detto a ridurre la funzione del medico, garantisce nel contempo un ruolo importantissimo a chi governa la comunità. È l'abate, infatti, che in tutte le regole e in tutte le realtà monastiche è detentore di un'autorità assoluta, che valuta la sincerità del monaco che si dichiara malato, che stabilisce se questi abbia diritto o meno al ricovero in infermeria (con i privilegi connessi). È lui che dà indicazioni dietetiche e perfino l'autorizzazione al bagno. È lui che ha potere discrezionale e derogatorio rispetto a quanto stabilito in consuetudini e regole. Tali poteri sono quelli che garantiscono l'ordinato svolgimento della vita comune, che consentono il governo delle diversità, inevitabili in una comunità, ma soprattutto le ineguaglianze, siano esse sociali, di età, nonché quelle, appunto, determinate dallo stato di salute. Accade infatti che il malato venga guardato dai confratelli con invidia e con ostilità (a volte addirittura insultato) perché le cure di cui necessita riducono o limitano temporaneamente la durezza della vita monastica. Per questo, le cure necessarie ad affrontare le varie infermità e soprattutto la dieta - calibrata sulle necessità individuali (cibi cotti o crudi, caldi o freddi) e senza dubbio influenzata dalle conoscenze mediche correnti anche se non sempre messa in relazione con i vari disturbi fanno capo all'autorità del superiore, perché possono (così come eventuali esenzioni dal lavoro o dagli obblighi liturgici) scatenare un diffuso disordine ${ }^{32}$.

Nella complessa e affollata realtà di questi primi monasteri, pare fondamentale insomma evitare i rischi insiti nella simulazione e nella rilassatezza, entrambe nemiche, oltre che della salute dell'anima del singolo, della efficiente organizzazione sociale ed economica del monastero, in cui il lavoro e il commercio della produzione sono aspetti tutt'altro che secondari ${ }^{3}$. Si nota insomma una forte dimensione "sociale", non tanto medica, nel controllo della malattia. È dunque da questo bisogno di non compromettere la dimensione omologante e standardizzata della vita monastica che nasce il bisogno di disporre di locali appositi per i malati il cui scopo è anche quello di sottrarre il paziente dalla vista (e dal sospetto) dei confratelli34. Ciò è ben percepibile laddove si dice che chi mormora e si lamenta per la fatica della vita monastica viene corretto per cinque volte e se nonostante questo, pur essendo adulto, disobbedirà, verrà condotto in infermeria dove resterà inattivo donec redeat ad veritatem ${ }^{35}$; come si vede si tratta di un rimedio disciplinare-spirituale, un tentativo di risoluzione del problema del tutto empirico basato sulla persuasione e sulla buona disposizione del sedicente malato.

Anche per quanto riguarda l'uso di farmaci per risolvere malattie accertate, non vi sono novità evidenti. La tradizione vigente all'esterno del monastero, come si è detto, fa la sua

\footnotetext{
${ }_{29}$ CRISLIP, op. cit. (n. 24), p. 19. Così a Shenoute e nel monastero pacomiamo governato da Horsiese, successore di Pacomio. La valutazione avviene nel refettorio, quando il monaco mostra di non essere in grado di mangiare in esso. La simulazione doveva essere frequente e l'intervento del superiore, illuminato da Cristo, doveva, con la sua autorità, evitare accuse reciproche ed insulti ai presunti simulatori. La facoltà del monaco di distinguere le varie afflizioni causate da demoni è sostenuta da Evagrio del Ponto, i cui influssi, peraltro, sono ben percepibili, in Occidente, negli scritti di Giovanni Cassiano, che riconosce agli asceti egiziani la conoscenza dei vizi che affliggono il monaco, la loro origine e la loro prevenzione; cfr. Cassiano, De cenobiorum institutis, PL, 49, libro IX, cap. XVI. Sempre nel libro IX si fa una attenta analisi della tristezza (col. $351 \mathrm{sgg}$.) che nel cap. XIII (col. 360) è definita passio, mentre nel cap. X (col. 360 sgg.) si tratta della accidia, per la definizione e il superamento della quale non si esita a ricorrere a metafore medico chirurgiche e a individuarne il rimedio nel lavoro (ibidem, cap. VIII, col 376), definito da san Paolo, spiritalis medicus (II Thess., III).

${ }^{30}$ D'altronde anche papa Gregorio Magno, nella sua Regula pastoralis, ovviamente non rivolta a monaci, paragona metaforicamente la malattia (non fisica) al peccato: è zoppo chi vede ciò cui deve tendere, ma non tiene la retta via della vita «per infirmitatem mentis» (GREGORIO MAGNO, Regulae pastoralis liber, PL, 1. I, col. 13-128).

${ }^{31}$ Ad monachos Magistri Regula, PL, 88, col. 943-1052, cap. 69 (De fratribus aegrotis), col. 1023-1024.

${ }^{32}$ CRISLIP, op. cit. (n. 24), p. 29. Forte è anche il controllo delle visite di esterni e familiari al malato. Ciò dimostra che il ruolo della famiglia non è totalmente superato, ma rigorosamente mediato dal monastero. Nella traduzione latina della regola pacomiana (Translatio latina Regulae sancti Pachomii, PL, vol. 23, p. 67-82) si contempla che nessuno entri in infermeria se non è malato mentre chi si ammala viene portato del preposito nel refettorio dei malati e il preposito si mette in contatto con gli infermieri che dispensano ciò che è necessario in vestiti o cibo (cap. 42). Il malato, così isolato, non deve entrare nel refettorio e mangiare ciò che vuole, né può portare in cella niente dalla infermeria, neppure un frutto (cap. 43). La visita al malato, neppure quella dei parenti, è possibile solo con il permesso del superiore (cap. 47), mentre i cibi portati al malato dall'esterno, pur non proibiti, sono ritirati, se di companatico si tratta, dal portinaio che li porta all'infermeria e non esclusivamente al malato cui sono destinati; se invece si tratta di dolci o frutta, il portinaio ne dà al malato quanto serve e il resto viene consegnato agli infermieri (cap. 53). I bagni sono vietati se il male non è grave, così come l'ungitura di tutto il corpo (cap. 92). Il malato però non è esente dall'obbligo di presenziare alle sepolture aiutato e sorretto da un infermiere (cap. 129).

33 Per questo, vd. CRISLIP, op. cit. (n. 24), passim. Il lavoro è facilmente connesso alla malattia. Nella regola pacomiana i due concetti sono presenti laddove si parla della pigrizia, che non è tollerata e che conduce l'uomo alla vergogna e alla mendicità. Non può essere definita pigrizia, invece, l'inattività del malato e del vecchio i quali possono trovare chi lavori per loro e allora avranno diritto al pane e a restare in portineria (quindi separati dai confratelli attivi), per un periodo ragionevole che non si configuri come sfruttamento della disponibilità dei confratelli. I malati godono di alcuni privilegi; ad esempio le focacce sono destinate all'infermeria e quando ci sono piccoli pani 5 ceste sono riservate all'infermeria perché i malati ne abbiano una razione a parte, sempre sotto la decisione del preposito; cfr. Pacomio e i suoi discepoli. Regole e scritti, a cura di L. Cremaschi, Magnano 1988, n. 18.

${ }_{34}$ PACOMIO, Regula a Hieronymo in latinum sermonem conversam, PL, 50, col. 271-304, cap. 20, col. 282 (Nullus introeat locum aegrotantium qui non aegrotat...).

${ }_{35}$ Praecepta atque judicia s.p.n. Pachomii, PL, 23, col. 82-92, cap. 164, col. 86 (Qui habet consuetudinem murmurandi...).
} 
comparsa sia nel contesto egiziano ${ }^{36}$, sia nel Mediterraneo di lingua greca, sia nei monasteri basiliani di Cappadocia, in cui, pur in assenza di relazioni precise (come già si è detto nel caso della dieta) tra erbe curative e malattia, essa viene inquadrata entro convinzioni religiose: al pari della medicina (quando accettata) si ritiene infatti che le erbe siano state dispensate da Dio all'umanità come conforto ${ }^{37}$. Il legame con la tradizione galenica greca, a cui si aprirà anche il monachesimo occidentale è evidente ${ }^{38}$. Ma considerazioni analoghe potrebbero farsi anche per il ricorso alla chirurgia, cui si è accennato.

La concezione della cura e dell'assistenza presente nel monachesimo basiliano si proietta nelle regole occidentali e poi soprattutto in quella di Benedetto. Anche qui i religiosi, nella maggiore o minore conoscenza e considerazione della scienza, tengono la medicina a dovuta distanza, accettandone ciò che è compatibile con la concezione religiosa della vita monastica e riservandosi la possibilità di decidere se, quanto e quando essa sia utile, nell'interesse generale e nella realistica consapevolezza delle deboli forze del monaco che, nel momento in cui provenga da ceti sociali alti, risulta più sensibile alle fatiche della vita ascetica e comunitaria e più propenso a ricorrere ai rimedi proposti dalla medicina. Ma il cenobio non si muove solo sul piano della realtà con le sue inevitabili dinamiche e non perde la sua funzione originaria di strumento utile al raggiungimento di un alto ideale di vita religiosa; la malattia, pertanto, nel solco della tradizione già risalente alle antiche esperienze di asceti e anacoreti, continua a rappresentare un momento importante per comprendere le trasformazioni della sensibilità religiosa entro contesti lontani per tradizione, per cultura, per organizzazione della società. Non v'è dubbio che un punto di osservazione significativo sia la Regola di san Benedetto la cui portata innovativa si può cogliere anche nei passi dedicati alla malattia, se confrontati, come ha fatto de Vogüé, con i testi delle regole di Basilio e di Agostino oltre che con la Regola del Maestro.

È impossibile soffermarsi nel dettaglio su questo confronto plurimo, che arriva a conclusioni chiare e importanti. Il focus deve essere quello del cap. 36 della Regola di Benedetto, che è articolata in due momenti: il primo di natura più teorica e spirituale e il secondo di natura più organizzativa e pratica. Sul primo punto è possibile notare le innovazioni di Benedetto; infatti, mentre la Regula Magistri, nei capp. 69 (De fratribus aegrotis) e 70 (De charitate fratrum circa aegrotos), cita il precetto evangelico per sollecitare generi- camente gli atti di spontanea carità nei confronti dei malati, un invito alla pratica caritatevole insomma ${ }^{39}$, nel testo di Benedetto, la carità nei confronti dei malati viene prima di tutto, dal momento che il servizio al malato è equiparato al servizio prestato a Cristo stesso. Il valore, indiscutibile, dell'azione caritatevole, dello zelo individuale è insomma superato dalla grande dignità dell'atto indicato come dovere collettivo e assurto a principio teorico. Per questo, l'ispirazione di Benedetto va ricercata più che nella Regula Magistri in Basilio, soprattutto quando l'uno e l'altro parlano del rapporto che intercorre tra malato e chi lo assiste servendolo. Ma Benedetto va anche oltre Basilio. Quest'ultimo si rivolge infatti alla responsabilità dei superiori che devono vegliare sulla salute morale dei monaci e quindi anche del malato, che deve avere un atteggiamento rispettoso e conveniente. Benedetto, invece, si rivolge direttamente al malato, con ciò prefigurando un rapporto assai più responsabile, intimo e biunivoco con chi lo assiste: il malato deve meritare l'attenzione del fratello evitando di tormentarlo con vane richieste, facilitando addirittura nel fratello sano la possibilità di ravvisare, in lui bisognoso, Cristo stesso ${ }^{40}$.

Questo legame in Cristo va dunque ben oltre il generico dovere di praticare la carità verso chi versa in stato di necessità; la vicinanza amorevole, la pazienza e la capacità di sopportazione, il fine che accomuna chi riceve e chi dona diventano potenti strumenti di tranquilla ascesi quotidiana per entrambi. Nello stesso tempo, assai realisticamente, viene tuttavia contemplata anche la difficoltà insita in questo alto ideale, sempre nell'interesse primario della salute spirituale del monaco. Infatti, nel caso in cui l'ardua prova della malattia non consenta al malato di instaurare un virtuoso rapporto con il fratello che lo assiste, quest'ultimo dovrà comunque trattarlo pazientemente, guadagnando con ciò un più alto livello di perfezione e una maggiore ricompensa. Tanta disponibilità non può derivare solo dall'accettazione di una imposizione (per quanto autorevole) di chi governa la comunità monastica, ma solo da un'intima convinzione che la Regola e la meditazione sulla Regola devono suscitare. In questo orizzonte di pensiero cadono i forti e reiterati sospetti di simulazione nei confronti di chi lamenta malattie non fisiche: un altro segnale di quanto Benedetto si distacchi da Basilio, che nell'Asceticon prevedeva per il fratello non veritiero la repressione del superiore e infine l'espulsione dal monastero ${ }^{41}$, ed anche dalla Regula Magistri in cui permanevano tracce della severità organizzativa dei cenobi orientali ${ }^{42}$.

\footnotetext{
${ }^{36}$ A Shenoute si distinguono disturbi interni e nascosti, mal di testa, di addome, di viscere, ecc., mentre nel cenobio di Apa Geremia in Saqqara frammenti di un manoscritto farmaceutico copto, di un erbario, di una iscrizione dell'infermeria restituiscono l'uso, per particolari patologie, di poltiglie d'erbe, di carbone tritato, di grasso animale, di escrementi (per l'apparato digestivo); cfr. CRISLIP, op. cit. (n. 24).

37 BASILIO, Regulae fisius tractatae, op. cit. (n. 14), cap. 55.

${ }^{38}$ Cassiodoro, in Italia, raccomanda ai medici monastici il ricorso a rimedi simili (CASSIODORO, De institutione divinarum lietterarum, PL, 70, col. 11051150, cap. 31 (De monachis curam infirmorum habentibus, con riferimenti a Discoride, Ippocrate, Galeno).

39 Nella Regola del Maestro (I: Introduzione, traduzione e commento a cura di M. Bozzi; II: Introduzione, testo e note a cura di M. Bozzi e A. Grilli, Brescia 1965 [= Regola del Maestro]), al cap. XXVIII si parla anche del digiuno, da cui i malati sono esentati. Però proprio per questo i pasti di sani e malati sono separati; i primi mangiano alla sesta mentre i secondi alla terza. A nessun vincolo di orario sono tenuti gli ammalati gravi in procinto di morte. Anche in questo caso si temono simulazioni della malattia ma la regola ammette che la motivazione per l'esenzione dal digiuno è la debolezza (spiritus promptus, caro infirma, Matteo XXVI) dell'infermo, equiparabile a quella dell'infantulus e del vecchio. In questo caso si allude anche all'esenzione dal lavoro se gli infantuli (così si dice) in estate, quando la giornata è più lunga, hanno minore forza per la loro età.

${ }^{40}$ A. DE VOGÜE, La Règle de Saint Benoît, VI, Paris 1971 (Sources chrétiennes, 186, Série des textes monastiques d'Occident, n. XXXIX), p. 1080 sgg.

${ }^{41}$ Ibidem, p. 1086.

${ }_{42}$ Regola del Maestro, op. cit. (n. 39), cap. 69.
} 
Quanto alla seconda parte del capitolo della Regola benedettina, quello di contenuto più pratico e organizzativo, anche in questo caso il distacco con le precedenti regole è degno di nota. Mentre la Regula Magistri non si preoccupa di infermeria, di infermieri, di bagni e di diete a base di carne (forse per la ridotta dimensione della comunità cui si riferiva), Benedetto se ne occupa e anche in questo ci appare assai innovativo, pronto com'è ad ispirarsi alle regole precedenti (e dunque alla tradizione cenobitica orientale) quando si rivelassero più idonee a garantire una corretta convivenza di un consistente numero di monaci. Il luogo deputato al ricovero dei malati dei monasteri egiziani era soprattutto pensato per difendere, come si è detto, la vita regolare dei monaci sani ed attivi. Già con Cesario vediamo come l'interesse per il benessere dei malati aumenti; nella sua regola sono previsti una cella comune con cantina e cucina proprie e in questa direzione si muovono anche le regole di Isidoro ${ }^{43}$ e di Fruttuoso di Braga che conosce la realtà orientale ${ }^{44}$. Benedetto anche in questo caso va oltre ed esplicita l'idea di una sistemazione apposita (super se deputata) nell'interesse esclusivo dei malati, che con maggiore agio possono usufruire di un infermiere e del bagno. Proprio nei confronti del bagno, che Pacomio consentiva ai malati manifesti ma con molte cautele per cercare di contenere ogni sorta di sollecitazione sessuale (una preoccupazione che ritorna in Agostino, in Cassiodoro e in Isidoro, il quale tuttavia permetteva il bagno anche in caso di polluzione notturna), Benedetto ha un atteggiamento più moderato che consente al malato, sulla scia della Regula virginum di Agostino, di poterne usufruire quando necessario. Analoga liberalità è riservata ai bisogni alimentari e, nello specifico, al consumo di carne. Sulla scia della regola di Cesario, Benedetto, che non approva il consumo di carne in condizioni normali, lo riserva però ai malati e ai molto deboli ${ }^{45}$.

Insomma, su queste questioni pratiche Benedetto dimostra liberalità e soprattutto evita posizioni troppo rigide; il che apre la regola a possibili e sensate interpretazioni, mo- tivate dalla singolarità di situazioni e di consuetudini, che diventeranno preziose quando, nell'VIII secolo i monasteri occidentali si doteranno di hospitia per poveri e pellegrini in cui la funzione di foresteria, in realtà limitata, si sarebbe estesa, seppur per ragioni contingenti, a malati esterni bisognosi di cura ${ }^{46}$. Ma i pregi della regola benedettina, consistenti in una maggiore duttilità, diventeranno ancor più evidenti in età carolingia, quando la Regola avrà una forte diffusione ad opera di Benedetto di Aniane e dovrà confrontarsi con una sensibile varietà di tradizioni e di consuetudini. I commenti di Smaragdo di Saint-Mihiel e di Ildemaro di Corbie (IX secolo) lo comprovano. Smaragdo ${ }^{47}$ nel suo commento fornisce una lettura assai attenta agli aspetti di umana fraternità della Regola, in particolare anche in relazione alla malattia. Sulla visita agli infermi ribadisce che i «monachi debent se invicem visitare saepissime», esaltando dunque quel rapporto di reciprocità tra assistito e curante raccomandato dalla Regola di cui abbiamo parlato. Inoltre sottolinea come il concetto di infermo debba comprendere non solo il monaco aegrotus ma anche il monaco animo molestus et contristatus: la malattia fisica, dunque, ma anche quella spirituale cui si riserva una non minore attenzione ${ }^{4}$. Egli sa bene che la tribulatio del monaco a volte suscitatur a daemone e che l'infirmitas animae richiede un medico come l'infirmitas corporis ${ }^{49}$, sull'esempio della carità dell'abate nei confronti di chi pecca: d'altronde anche il peccato è equiparato all'infermità e il peccatore al malato che deve essere riportato alla salute e cioè al servizio del Creatore ${ }^{50}$. Da questo compito primario discendono azioni pratiche tese a mettere il malato a proprio agio per impedire che la malattia (così come qualsiasi altra deviazione) possa trasformarsi in una occasione per compromettere la scelta del monaco di restare lontano dalla società e dalla famiglia a suo tempo abbandonate ${ }^{51}$. Come si vede il fine ultimo sono le necessità spirituali, il rafforzamento della vocazione monastica, più che non il rispetto formale delle norme.

43 ISIDORO HISPALENSIS, Regula monachorum, PL, 83, col. 867-894, a col. 892, cap. XXI: De infirmis: la loro cura spetta a un monaco di sana e santa conversazione e devono assumere cibi delicati perché si ristabiliscano e possano tornare alla consuetudine. Anche in questo caso la cura è vista come possibile fonte di scandalo per i sani che hanno evidenti difficoltà a tollerare gli infermi e anche qui si allude a casi di finzione della malattia.

${ }^{44}$ FRUCTUOSUS BRANCARENSIS EPISCOPUS, Regula monachorum, PL, 87, col. 1097-1130, in cui sembrano previste assistenza e cura anche a non monaci. Gli infermi sono trattati, insieme agli ospiti e ai pellegrini, con moderazione e compassione e sono oggetto delle cure di ministri che preparano loro le vivande (cap. 5: De mensis e cap. 10: De hospitibus, peregrinis et infirmis). Si parla infatti di uomini che vanno in monastero con moglie e figli piccoli (al di sotto dei 7 anni) e che sono soggetti alla potestà dell'abate e è il cellarius, esonerato dai servizi, che se ne occupa (cap. 6: Qualiter debeant viri cum uxoribus ac filiis absque periculo vivere in monasterio); qualora malati, stiano «in una domo» assistiti da un monaco a ciò delegato (prepositus), oltre che dal cellarius, e ciò perché non ricerchino le cure dei parenti e le delizie della città (cap. 7: Qualiter infirmi in monasterio deveant teneri). I malati devono essere discreti, ilari, riconoscenti a Dio, mentre chi li assiste non deve offenderli. I monaci malati continuano a stare nel monastero ma anch'essi, a maggior ragione, non possono essere assistiti da esterni. Qualora una donna volesse fare loro visita, dovrà essere accompagnata dal ministro, ma senza trattenersi (cap. 17: Qualis debeat esse consuetudo salutandi in monasteriis virorum puellarumve). Sui rischi della cura al di fuori del monastero, vd. anche le regole di Lérins e in particolare la III Patrum Regula ad monachos (V sec.), PL, 103, col. 443-446, al cap. XII («Id etiam pro custodienda fama specialiter statuimus, ut nullus monachus in infirmitate positus relicto monasterio parentum suorum studio commendetur: quia magis eum saecularium spectaculorum visu aut auditu pollui censemus, quam ab aegrtudine posse purgari»); per il collegamento di questa regola con Lérins, vd. A. De Vogüé, Introduction a Les regles des saints pères, 2 voll. (Sources chrétiennes, 297, 298), Paris 1982.

${ }^{45}$ Per questi aspetti alimentari, la proibizione e l'uso medico-dietetico della carne, cfr. ARCHETTI, Mensura victus constituere, op. cit. (n. 28 ), p. 780-792.

${ }^{46}$ I. GOBRY, G. SPINELLI, L'Europa di Cluny. Riforme monastiche e società d'Occidente: sec. VIII-XI, Milano 1999, p. 439

${ }^{47}$ Smaragdo, Commentaria in regulam Sancti Benedicti, PL, 102, col. 689-932.

${ }^{48}$ Ibidem, cap. 16, col. 759 (Infirmum visitare).

49 Ibidem, cap. 18-19, col. 760 (In tribulatione subvenire).

${ }^{50}$ Ibidem, cap. 27, col. 852 (Qualiter debeat esse sollicitus abbas circa excommunicatos).

${ }^{51}$ Ibidem, cap. 36, col. 868 (De infirmis fratribus): l'abate deve garantire una cella separata ai malati cum omnibus oportunitatibus ut nullum exteriorem laborem sentiant qui in infirma carne poenam portant e il cellerarius e il praepositus provvedano alle sue necessità perché i malati nec propinquorum affectus nec urbium delicias requirant. Tutte queste attenzioni sono tenute a maggior ragione presenti nel caso di vecchi e bambini (Ibidem, cap. 37, col. 870: De senibus vel infantibus). 
Passando a Ildemaro ${ }^{22}$ varrà la pena soffermarsi brevemente, anche in questo caso, sul commento al solito cap. 36 della Regola (De infirmis fratribus) ${ }^{53}$ in cui si dice addirittura che «nulla res ita agenda est cum tanto studio quanto domus infirmorum»; tanta preoccupazione e tanta enfasi è equiparabile a quella riservata alla custodia infantum. Ebbene, il luogo della malattia diventa centrale e funzionale al compito spirituale del servizio ai malati, in tutto equiparabile al servizio di Cristo. Il monastero deve pertanto impegnarsi seriamente nell'organizzazione dell'assistenza al malato $(\mathrm{ad}$ domum, ad focum, ad victum atque potum et cibum et omnia necessaria infirmorum), in modo da non suscitare nel monaco (nei confronti del quale l'attenzione rimane altissima) ira o murmuratio proprio alla fine della vita, quando la vittoria e il premio sono vicini: un timore di disperdere il patrimonio spirituale accumulato grazie alla scelta monastica (di cui la malattia è una sorta di coronamento) che sembra l'eco del consiglio di Antonio ad Eulogio di cui si è detto.

Gli infermi devono dunque avere, secondo Benedetto, una cella super se deputata e un servitore timorato di Dio, diligente e sollecito. E Ildemaro specifica che col termine cella la Regola non intende parlare de una mansione e cioè di un unico locale, sed de claustra, cioè di una domus con tante mansiones. Infatti in una sola mansio - dice Ildemaro - come possono stare senza disturbarsi a vicenda, quattro fratres di cui uno moribondo, un altro che vomita, un altro che vuole mangiare e un altro ancora che deve liberarsi? Per tale domus infirmorum, dunque, si prospetta una sempre più ampia e complessa articolazione: essa deve avere nelle sue vicinanze un oratorio che garantisca ai malati allettati la possibilità di sentire messa e comunicarsi e una cella appositamente deputata ad ospitare l'abate quando è malato, sufficientemente autonoma in modo da consentire a quest'ultimo di ricevere e di parlare con gli ospiti senza disturbare gli infermi. Del servitore, poi, si elencano e si precisano le qualità partendo dall'analisi dei termini che nella Regola lo designano: timens Deum, diligens, sollicitus. Il termine sollicitus rimanda tanto alla sapienza e alla cautela nel trattare il malato facilmente irascibile, quanto alla forza (possibilitas) indispensabile per sollevare e trasportare il malato, nonché allo zelo cioè all'amore per il prossimo inteso come servizio svolto per amore di Dio. Le numerose qualità del servitore, che deve essere monaco, sembrano implicare una qualche forma di selezione tra i fratelli demandata al discernimento del priore, sempre nell'interesse fisico e spirituale dei malati; ma soprattutto indicano che l'assistenza, proprio per il suo profondo significato spirituale, è rigorosamente riservata ai confratelli. Laici o canonici possono essere destinati, invece, a funzioni quali il lavaggio dei vestiti sporchi del malato o al rifornimento di carni per il suo sostentamento: il che sottende, ancora una volta, una struttura di supporto non banale alle esigenze della domus infirmorum. E nei confronti del malato l'abate, cui tutto compete, deve, con una corretta e autorevole interpretazione della regola, sapere leggere dalle corporis actiones (pigrizia all'ufficio, sonnolenza, negligenza nelle opere) i segnali dell'infermità dell'anima quasi ne fosse il medico: «quia sicut corpus habet infirmitates suas, id est febrem et cetera similia, ita habet anima suas infirmitates, id est iram, furorem, indignationem etc. et quantum plus opera negligenter facta tanto ex maiore infirmitate animae descendunt».

Anche sul bagno al malato e sull'uso della carne, Ildemaro, nel suo commento, sfrutta appieno la duttilità della Regola: i malati possono lavarsi anche due volte al giorno e quando ve ne sia bisogno, mentre la carne è consentita perfino ai moribondi e non solo a coloro che devono riprendersi ${ }^{4}$. Infatti è necessario tenere conto (soprattutto ai fini della dieta) delle differenze tra malato e malato e soprattutto tra i debiles e gli aegroti: mentre i secondi sono afflitti da malattia grave e addirittura allettati, i primi sono da intendersi non solo gli infantes o i senes, più soggetti a malattie e più facilmente provati dalle fatiche della vita monastica, ma anche i maiores iuvenes qui pro aliqua incommoditate corporis, qui aut pro nimio calore, aut pro spasmo, vel pro aliqua incommoditate fiunt a semetipsis debiles. Tale significativo allargamento delle tipologie di infermità ha delle conseguenze anche sul piano delle proibizioni alimentari, che devono tenere conto delle consuetudini locali, dal momento che brusche variazioni possono generare ulteriori disturbi. Ildemaro, così, ricorda che in Francia non si dà carne a chi giace a letto, ma solo a chi comincia ad alzarsi perché riacquisti forza; quando poi il malato lascia l'infermeria e torna al refettorio, il cellerario prepara per lui pesci o cibi più delicati per alcuni giorni finché non sia in grado di tornare alla alimentazione normale prevista dalla Regola. Questa attenta valutazione della singolarità dei casi determina anche la mensura potus che, dovendo oltretutto tenere conto della varietà regionale dell'unità di misura $($ mina $)$, non può essere normata una volta per tutte ${ }^{55}$. Varietà e spazi di manovra erano dunque possibili e la Regola non pretendeva di condannarli e sradicarli. D’altronde un esempio di saggia organizzazione monastica ci è restituito anche dalla Vita di

\footnotetext{
${ }^{52}$ R. MITTERMÜLLER (a cura di), Vita et Regula Ss. p. Benedicti una cum expositione Regulae, III: Expositio Regulae ab Hildemaro tradita et nunc primum typis mandata, Ratisbona 188o. Su Ildemaro e sull'importanza del suo commento, vd. G. ARCHETTI, Ildemaro a Brescia e la pedagogia monastica nel commento alla Regola, in San Faustino Maggiore di Brescia: il monastero della città, Atti della giornata nazionale di studio (Brescia, Università Cattolica del Sacro Cuore, 11 febbraio 2005), a c. di G. Archetti, A. Baronio, Brescia 2006 (Brixia Sacra. Memorie storiche della diocesi di Brescia, XI, 1), pp. 113-123 con i relativi rimandi.

${ }^{53}$ Expositio Regulae ab Hildemaro tradita, cap. 36, pp. 403 sgg.; in proposito, G. ARCHETTI, "Dilexi decorem domus tuae". Committenza aristocratica e popolare in ambito claustrale (secoli VIII-XII), in Il Medioevo: $i$ committenti, Atti del Convegno internazionale di studi (Parma, 21-26 settembre 2010), a cura di A. C. Quintavalle, Milano 2011 (I convegni di Parma, 13), pp. 244-246; ID., Spazi e strutture claustrali nei commenti carolingi alla Regola benedettina, «Hortus artium medievalium», 20/2 (2014), pp. 448-462, mentre per un confronto con le strutture di accoglienza, secondo il commentatore carolingio e la regola benedettina, ID., Pellegrini e ospitalità nel medioevo. Dalla storiografia locale all'ospedale di Santa Giulia di Brescia, in Lungo le strade della fede. Pellegrini e pellegrinaggio nel Bresciano, Atti della Giornata di studio (Brescia, 16 dicembre 200o), a cura di G. Archetti, Brescia 2001 (Brixia sacra. Memorie storiche della diocesi di Brescia, VI, 3-4), pp. 83-104.

${ }^{54}$ In proposito si rimanda ad ARCHETTI, "Infundit vinum et oleum”, op. cit. (n. 28), pp. 1120-1136; ID., Mensura victus constituere, op. cit. (n. 28 ), pp. 780-792.

55 Expositio Regulae ab Hildemaro tradita, op. cit. (n. 52), cap. XL, p. 443; per un commento si rimanda ad ARCHETTI, "Infundit vinum et oleum", op. cit. (n. 28), pp. 1136-1203; ID., "Il vino non è per i monaci". Appunti sparsi sugli usi monastici antichi, in "Dulcius nil est mihi veritate". Studi in onore di Pasquale Corsi, a cura di F. Monteleone e L. Lofoco, Foggia 2015, pp. 65-87.
} 
Eugendo laddove si dice che il santo abate, oltre ad opporsi agli usi orientali eliminando le dimore singole dei monaci e preferendo ambienti comuni "specializzati" per funzione (xenodochio, refettorio, dormitorio), si preoccupava molto dei malati che potevano scegliere sia coloro che li dovevano curare, sia la dieta ritenuta più acconcia ${ }^{56}$.

L'idea monastica della malattia e della cura emerge anche, pur nella diversità dei contesti e della tipologia delle testimonianze, nei casi in cui si narra di non-monaci che guardano con speranza al monastero e ai santi padri che lo abitano nella speranza di una guarigione che trascenda la limitatezza dell'arte medica. E si tratta di una speranza che spesso non si esaurisce con la morte del monaco/guaritore/santo, ma si prolunga oltre la vita di quest'ultimo grazie al potere salutifero di reliquie e sepolture che danno lustro al monastero in cui sono devotamente conservate. In età merovingia ad esempio, anche per la contiguità del monachesimo al contesto politico asserita dal Prinz ${ }^{57}$, le narrazioni agiografiche indugiano sui casi di guarigioni miracolose - presenti nella storia monastica fin dalle origini - enfatizzando i rapporti tra monastero e mondo esterno, rappresentato quest'ultimo soprattutto dai ceti alti e da personaggi di rango. La Vita Ursmari episcopi et abbatis Lobbiensis, ad esempio, narra di una nipote di Ursmaro accolta nel monastero e commendata a Santa Aldegunda che, diventata adulta, manifesta la malattia al collo «que vulgo dicitur guttreria». Ursmaro non esita a consultare il medico che propone un doloroso intervento chirurgico, ma il parere del medico non viene tenuto in conto. Ursmaro, infatti, non vuole che la nipote soffra e preferisce riporre tutta la fiducia della guarigione in Dio con ciò escludendo l'arte medica dalla sfera monastica ${ }^{58}$.

Nella Vita di Severino abate di San Maurizio di Agauno, composta pare nella prima metà del IX secolo da Fausto, discepolo di Severino, si descrive la malattia di Clodoveo, affetto da un freddo che non lo abbandona per due anni. In domo regis non manca Tranquillinus doctor omni sapientia plenus honores arte medicine gerens; ma in questo caso è il medico stesso che dichiara la sua impotenza e, suggerendo una soluzione che ne arretra spontaneamente il campo di azione, consiglia al re di andare al monastero di Agauno dove giace il beato martire Maurizio e dove c'è un santo uomo (Severino appunto), che guarisce molti da malattie fisiche e demoniache con le sue preghiere ${ }^{59}$. Ė un percorso, questo, che conduce fino a Cluny, il cui abate Maiolo guarisce con mezzi sacrali non solo personaggi di alto rango, ma anche il ricco monetario pavese Ildebrando che, dopo aver speso molto in medici (oltre che in pie offerte), avendo appresa la notizia del passaggio dell'abate nella sua città, lo incontra e da lui ottiene guarigione ${ }^{60}$.

La medicina e i medici dunque esistono, ma la concezione della malattia maturata in ambito monastico fa sì che l'una e gli altri vengano presentati come sottoposti ad un potere superiore che oltretutto presenta maggiori vantaggi rispetto ai rimedi proposti dal sapere professionale, contribuendo ad un vantaggioso avvicinamento del potere alla dimensione sacrale. Ne consegue l'apparente stranezza che gli autori delle Vite dei santi possono ricorrere da un lato ad un linguaggio medico per affrontare questioni di correzione morale (con riferimenti anche alla chirurgia e ai farmaci) e dall'altro evocare il potere terapeutico soprannaturale del monaco per guarire vari tipi di malattie, non solo spirituali o generate da presenze demoniache ma anche fisiche ${ }^{61}$.

A ben guardare, queste interferenze equeste sollecitazioni contribuiscono a fare del monastero il luogo in cui cura e assistenza possono ritagliarsi spazi privilegiati e a recepire duttilmente le trasformazioni culturali. Non ci stupiremo pertanto se nella Vita Galli di Walafrido ci appare un frater quidam eiusdem congregationis medicinali scientia non ignobiliter instructus che pratica flebotomie ${ }^{62}$. Così pure alcuni manoscritti di area germanica documentano le cognizioni mediche di Iso († 871) e Notkero II († 975), mentre le fonti dell'abbazia di San Gallo ricordano monaci e maestri che fanno di essa nel IX e X secolo la sede di studio più importante di Oltralpe. Sono proprio gli studi codicologici che ci portano a ravvisare in altri monasteri luoghi in cui si conservano e si depositano le tracce della scienza antica: in questo senso l'Italia può avere costituito un tramite per la diffusione di un sapere medico in ambito monastico se pensiamo a Nonantola eai suoi manoscritti contenenti testi di Ippocrate, di Apuleio, di Celso, e altri riconducibili al suo abate Filagato, greco di Calabria vicino a Ottone III ${ }^{63}$.

Su una varietà di situazioni, di interpretazioni, di commentari (e quindi di consuetudini) collegabili alla duttilità della Regola benedettina eal potere discrezionale dell'abate, su certe esemplari aperture verso la cultura medica, vediamo tuttavia imporsi le Consuetudines di Cluny, che a loro volta avrebbero ispirato altri abati e altri monasteri. Guardando a queste fonti normative, si nota un atteggiamento duro e severo nei confronti del malato e una tendenza a rinchiudere la malattia, e i modi di affrontarla, entro una concezione fortemente collegata all'exitus vitae del monaco. L'attenzione pare meno rivolta alla cura del malato e all'intrinseco valore spirituale del rapporto tra infermo e curante; tende piuttosto a risolversi in formalità che antepongono la morte alla malattia, che della morte diventa il duro momento preparatorio. Il Liber tramitis aevi Odilonis abbatis (cap. 52: De infirmis fratribus) ci tramanda ad esempio l'esistenza di una domus infirmorum dove si dorme e si mangia separatamente e in cui la carne è rigorosamente proibita. I malati sono accuditi in momenti propri anche dal punto di vista religioso-liturgico e si vogliono rigidamente divisi dal conventus dei confratelli. Di notte sono obbligati ad alzarsi per

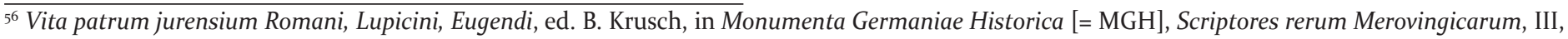
Hannoverae 1896 , p. 125- 166, a p. 164.

${ }^{57} \mathrm{~F}$. PRINZ, La presenza del monachesimo nella vita economica e sociale, in Dall'eremo al cenobio: la civiltà monastica in Italia dalle origini all'età di Dante, a c. di G.C. Alessio, Milano 1987, pp. 241-276; anche ID., Ascesi e cultura. Il monachesimo benedettino nel Medioevo, Roma-Bari 1983.

${ }^{58}$ Vita Ursmari episcopi et abbatis Lobbiensis, ed. B. Krusch et W. Levison, MGH, Scriptores rerum Merovingicarum, VI, Hannoverae 1910, pp. $453-461$, a p. 459.

59 Vita Severini abatis Acaunensis, ed. B. Krusch, MGH, Scriptores rerum Merovingicarum, III, Hannoverae 1904, p. 166-170, a p. 169-70.

${ }^{60}$ SIRO DI CLUNY, Maiolo abate di Cluny papa mancato, a cura di G. Spinelli e D. Tuniz, Milano 1994, p. 85.

${ }^{61}$ Vita patrum jurensium Romani, Lupicini, Eugendi, op. cit. (n. 56), p. 147. Si parla anche di una fanciulla di alta condizione sociale che, assalita da un demonio atroce, è claustris retrusa verum etiam ferrei vinculis tenebatur abstricta prima che il caso venisse risolto tramite esorcismo (ibidem, p. 158).

${ }^{62}$ Vita Galli confessoris triplex (auctore Valhafrido), ed. B. Krusch, MGH, Scriptores Rerum Merovingicarum, IV, Hannoverae 1904, pp. $280-337$.

${ }_{63}$ A. BECCARIA, I codici di medicina del periodo presalernitano (sec. IX-X-XI), Roma,1956, pp. 54 sgg., 62.
} 
l'ufficio e, se le forze non li sostengono, vengono comunque portati dai più giovani nell'oratorio, nella esplicita speranza che muoiano in Chiesa, come in effetti spesso accadeva ${ }^{64}$. Anche le consuetudini cluniacensi di Ulrico di Zell destinate ad Hirsau ci mostrano che malato e malattia erano inseriti in una organizzazione complessa, attentissima soprattutto alla dimensione liturgica e imbrigliata in una ritualità esasperata $^{65}$. La separazione del malato dalla quotidianità del monaco sano è ottenuta tramite forme di controllo quasi poliziesco nei locali deputati ai malati e prevede punizioni per il mancato rispetto delle norme ${ }^{66}$. Avendo evocato questo clima, non mi pare fuori luogo concludere con un cenno al De visitatione infirmorum, un testo già attribuito ad Agostino ma da ascriversi invece a Balderico abate di Saint Pierre de Bourgeuil (XI-XII sec.), in cui si esalta la malattia (qualificata infirmitas medicinalis). In esso si intima al malato di essere non semplicemente paziente, ma addirittura lieto in modo che il gravamen corporale si trasformi in antidotum spiritua$l e$, mentre la visitatio è di fatto riservata solo al monaco ormai moribondo ${ }^{67}$. Un monaco moribondo la cui esasperazione può esplodere in aperta ribellione e che reclama, per così dire, il proprio diritto a tagliare il traguardo della vita libero dall'attenzione ossessiva dei confratelli. È ciò che viene descritto nel Cronicon Casinense (primi anni del XII) in cui si narra la morte del monaco Randiscius. Quando i confratelli iniziano, «ut moris est, eius animam omnipotenti Deo commendare», il moribondo non esita a zittirli: «Tacete, tacete. Numquid non auditis quante resonant laudes in celo? $\mathrm{Nu}-$ mquid pueros qui laudes decantant videtis? Per Deum, vos postulo ut sileatis mihique tam suavissimum cantum audire sinatis» ${ }^{68}$. Detto questo spirò. Siamo lontani dalla malattia che non dispera della guarigione e che si pone soprattutto come strumento, condiviso coi fratelli e con la comunità, di vivo perfezionamento spirituale in Cristo. Sembra insomma che nei monasteri l'attenzione alla malattia, già strumento, pur doloroso, di ascesi individuale e trasformata poi in una straordinaria occasione di amorevole perfezionamento spirituale condiviso, si sia progressivamente spostata sulla morte e sui suoi grevi e severi riti.

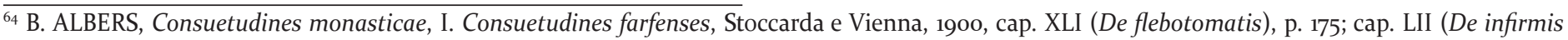
fratribus qualiter ab ipsis vel a ministrantibus eis agantur), p. 186; cap. LIII (Item infirmos fratres sic debent visere sodes), p. 189. Per queste consuetudini già ritenute spettanti a Farfa ma ora nuovamente riassegnate a Cluny, vd. Liber tramitis aevi Odilonis abbatis, ed. P. Dinter, Siegburg 1980 (Corpus consuetudinum monasticarum, X); inoltre, per il tema dell'infermità, R. CRISTIANI, "Infirmus sum, et non possum sequi conventum". L'esperienza della malattia nelle consuetudini cluniacensi dell’XI secolo, «Studi medievali», terza serie, XLI, 2 (2000), pp. 777-807.

${ }_{5}^{6}$ UDALRICUS CLUNIACENSIS MONACHUS, Antiquiores consuetudines Cluniacensis monasterii, PL, 149, col. 635- 778, 1. III, cap. XXV (De infirmario), col. 767; cap. XXVI (De famulis ad domum infirmorum pertinentibus), col. 769; cap. XXVII (De infirmis), col. 769; cap. XXVIII (De unctione cuiusque fratris), col. 779; cap. XXIX (Quid agendum est in fine exitus), col. 771. Sappiamo tuttavia che nell'XI secolo Guglielmo, abate di Hirsau si occupava degli indigenti, li visitava a domicilio, li seppelliva, curava i malati mentali e a volte li guariva; cfr. GOBRY, SPINELLI, L'Europa di Cluny, p. 437.

${ }^{66}$ Sui riti della morte a Cluny, vd. G. CANTARELLA, I monaci di Cluny, Torino 1993, p. 156 sgg.

${ }^{67}$ AGOSTINO (ma BALDRICUS DOLENSIS archiepiscopus), De visitatione infirmorum, PL, 40, col. 1147-1158, cap. VI, col. 1150 sg.

${ }^{68}$ Chronica monasterii Casinensis, a c. di H. Hoffmann, in MGH, Scriptores, XXXIV, Hannover 1980, p. 434.
} 